\title{
Active power filters - optimization of sizing and placement
}

\author{
M. MACIAZŻEK*, D. GRABOWSKI, and M. PASKO \\ Institute of Electrical Engineering and Computer Science, Silesian University of Technology, 10a Akademicka St., 44-100 Gliwice, Poland
}

\begin{abstract}
The significant problem of compensator placement and sizing in electrical networks has been analyzed in the paper. The compensation is usually realized by means of passive or active power filters. The former solution is widely used mainly because of the economical reasons, but the latter one becomes more and more popular as the number of nonlinear loads increases. Regardless of the compensator type the most important goal consists in voltage and current distortion drop below levels imposed by standards. Nevertheless, the desired effects should be achieved with the minimum cost. So far a few objective functions have been proposed for this optimization problem. It is claimed that minimization of the compensator currents leads also to the minimum costs. This paper shows that such simplified approach could lead to suboptimal solutions and in fact a function $g(\cdot)$ reflecting the relation between the compensator size and its price must be incorporated into objective functions. Moreover, in this case it is very easy to compare solutions obtained using compensators offered by different suppliers - it is enough to change the function $g(\cdot)$. Theoretical considerations have been illustrated by an example of active power filter allocation and sizing.
\end{abstract}

Key words: power quality, active power filter, harmonics, optimization.

\section{Introduction}

Transmission of electrical energy between sources and loads is usually carried out by means of three phase lines. Most of industrial loads belong to three phase loads, too. Voltages generated by three phase synchronous generators are sinusoidal and symmetrical. However, voltages as well as currents in a given power system cross-section are very often distorted due to widely used power electronic devices and large asymmetrical loads, e.g. arc furnaces, as well as a great number of low power loads, e.g. switched-mode power supplies, compact fluorescent lamps, which are nonlinear and non-stationary. Dynamic changes of a higher harmonic content in voltage and current time waveforms make resonance passive filters, currently used for distortion suppression, less effective. Significantly better results can be obtained when parallel or series active power filters (APF) [1], hybrid filters or UPFC (Unified Power Flow Controller) systems [2] are applied to limit higher harmonics. The optimal sizing and allocation of compensators have been considered for passive [3-6] and active or hybrid filters [7-15]. This paper presents a solution of the problem for active power filters.

The possibility of playing a role of the controlled current or voltage source is a distinctive feature of active power filters. Two basic types of APFs can be distinguished: parallel, which injects additional current, and series, which introduces extra voltage. Regardless of the filter type, the generated current or voltage lead to reduction of supplying voltage and current unwanted components, e.g. higher harmonics.

The aim of optimization usually consists in allocation of compensators having the minimum nominal currents which ensure the distortion drop below the limits indicated by standards $[11-13,15,16]$. The other approach consists in min- imization of the telephone interference factor (TIF), voltage total harmonic distortion (THD), harmonic transmission line losses or motor load losses, while keeping the compensator currents below the specified values [7-10, 17, 18]. Sometimes a multi-objective optimization approach is used in order to simultaneously minimize a few objective functions $[6,14$, $19,20]$.

Because the nominal current influences the compensator price, so in many works it has just been assumed that the optimization of compensator sizes leads also to the cost reduction and the economic goal is automatically taken into account [14, $16,20,21]$. This paper shows that such simplified approach could lead to suboptimal solutions. There are a few papers in which objective functions are directly based on investment costs $[6,11,15,19]$. However, contrary to the approach presented in this paper, the compensator cost is assumed to be a linear function of its size $[6,11,15,19]$.

Some new objective functions which enable optimal allocation and sizing of APFs in power systems have been proposed in [22]. They are an extension of the basic definitions used in other works, e.g. [8-10]. This paper includes the extension and verification of the ideas presented in [22].

The basic difference between research works carried out so far in the field consists not only in objective function definitions but also in algorithms used to solve the optimization problem. The number of optimization algorithms which can be used is very large. The choice depends among others on properties of the objective function, e.g. differentiability. First, GBDT algorithm [7] and combinatorial algorithms [23] were applied to solve the problem of compensator allocation and sizing. Later, TABU algorithm [11], modified adaptive or discrete particle swarm optimization $[6,14,15]$ and genetic algorithms $[8-10,19]$ have been applied. Lately, the particle

\footnotetext{
*e-mail: marcin.maciazek@polsl.pl
} 
swarm optimization algorithm has been combined with fuzzy approach and it seems to give very promising results [13]. In general, global optimization methods give the best results while solving the problem under consideration. This is the reason why genetic algorithms have been used in this paper.

Impact of the load impedance or capacitor bank changes which influence the overall frequency spectra of distorted waveforms in system nodes is usually not considered with exception to few authors who assume that the analysis is made for the worst case [7, 12]. In fact, for the supplying systems, in which the load variation cannot be neglected, the problem should be rather solved iteratively for successive steady states during the given time horizon [12].

In this paper it has been assumed that APFs can be controlled either by a classical or a modified algorithm. The former assumes that the APF injects currents, which cause the line currents at the point of installation to be as close to sinusoidal as possible [24] - local distortion reduction. The latter requires information about currents and voltages in all network nodes and enables global distortion reductions, i.e. in the entire network. The second approach is similar to network-wide harmonic reduction and cooperative control presented in [16, $18,21]$.
Methods of APF allocation, which ensure minimization of economical costs, are of great importance. High costs are one of the main reasons why in many applications passive resonance filters, which have worse characteristics but lower prices, are still used to eliminate higher harmonics. Constant deterioration of electrical power quality accompanied by increasing number of nonlinear loads makes research in this field essential. Electrical energy suppliers suffer more and more due to worsening of electrical power parameters either on their or customer side. Thus, power quality improvement is a crucial issue from the technical and economical point of view.

\section{Test system}

There are no Polish test systems which could be used for harmonic analysis in power systems. However, there are such test systems proposed by the IEEE group called Task Force on Harmonics Modeling and Simulation [25, 26]:

- 14-bus test system,

- 13-bus test system I,

- 13-bus test system II.

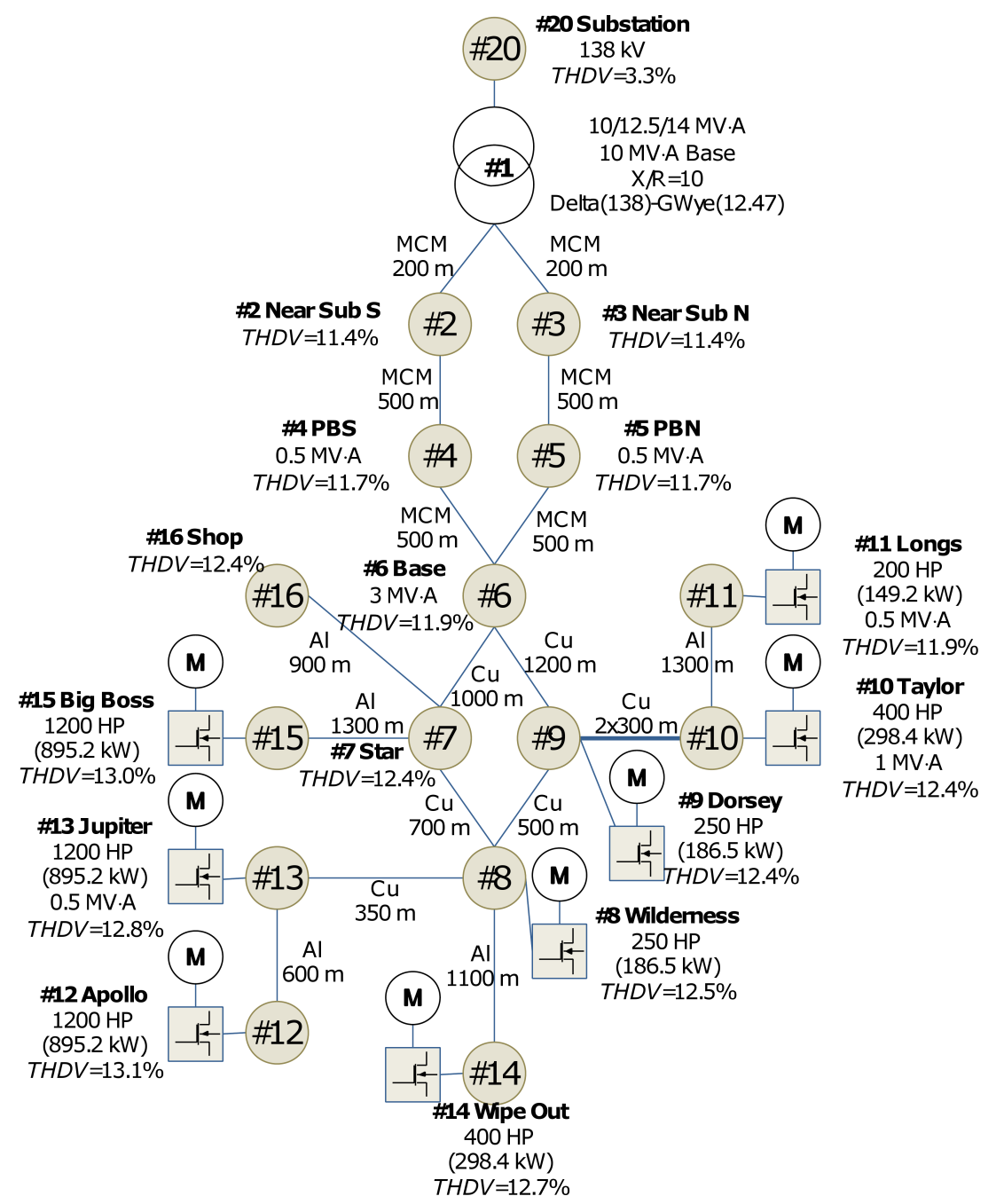

Fig. 1. Diagram of the test system 
Moreover, many models, developed by IEEE in order to enable power flow analysis in power systems, are available [27], e.g. IEEE 30-bus system, IEEE 57-bus system and even IEEE 300-bus system. Unfortunately, only parameters for the fundamental frequency are given for these systems and so their application in harmonic analysis requires some additional calculations. Moreover, they do not contain any nonlinear loads. These are the reasons why the system shown in Fig. 1 has been used as a test power system. This is a 17-bus system for ski area which has been described in [28]. It contains 8 buses which supply ski lifts powered by DC motors driven by six-pulse line-commutated adjustable speed drives (ASD). In Fig. 1 nominal powers of the motors are given in original units used in [28], i.e. in HP (Horse Power), $1 \mathrm{HP}=746 \mathrm{~W}$. Power electronics converters are the main source of harmonics in the analyzed power system. The system has been implemented in PCFLO software package [28] and THDV coefficients presented in Fig. 1 have been calculated just with the help of PCFLO. ed by:

The choice of the test system (Fig. 1) has been motivat-

- sufficiently large number of nonlinear loads being a source of higher harmonics and distributed in the system,

- distances between buses exceeding $1000 \mathrm{~m}$,

- complete technical data of the system, among others line model parameters, i.e. resistances $\mathrm{R}$ and reactances $\mathrm{X}$.

\section{Optimization strategies}

First, a goal function and some constraints have to be defined in order to solve the problem of optimum placement of higher harmonic compensators. Moreover, some assumptions are usually made when performing harmonic analysis of power systems, namely, that nonlinear loads can be regarded as current sources and the power system can be described by a linear model for each harmonic. So the impedance matrix describing the system can be determined separately for each frequency. An active power filter attached to any system bus is also modeled as an ideal current source injecting higher harmonics to the system.

The following three optimization strategies have been examined:

1. Minimization of THDI coefficients in buses to which APF are connected - the goal function $f_{A}$ :

$$
\begin{gathered}
\min _{\mathbf{x}} f_{A}(\mathbf{x})=\min _{\left\{\operatorname{Re}\left(I_{w h}^{k}\right), \operatorname{Im}\left(I_{w h}^{k}\right)\right\}} T H D I_{w}, \\
w=1,2, \ldots, W,
\end{gathered}
$$

where $w$ - bus number, $W$ - the number of APFs, $I_{w h}^{k}$ - phasor of the $h$ harmonic of the compensator current, $h=2, \ldots, H$.

2. Extension of the first strategy with the constraint put on THDV coefficients in all buses:

$$
T H D V_{w} \leq T H D V_{\max }, \quad w=1,2, . ., W^{\prime},
$$

where $W^{\prime}-$ the total number of buses.
This approach enables finding a solution which ensures limiting the $T H D V$ coefficients below the acceptable value $\left(T H D V_{\max }\right)$ defined in power quality standards - in this work the threshold has been assumed to be equal to $5 \%$ in accordance with [29].

3. Modification of the first strategy in order to take into account the economical criterion. It enables finding the optimum solution with respect to compensation cost and can be regarded as a compromise between power quality improvement and financial expenses required to reach this improvement. In this case the function $g(\cdot)$ which reflects the relation between the cost of a single compensator and its nominal current must be taken into account:

$$
\min _{\mathbf{x}} f_{C}(\mathbf{x})=\min _{i} \frac{\max _{w} T H D V_{w}^{[i+1]}-\max _{w} T H D V_{w}^{[i]}}{g\left(\left|I_{w}^{k}\right|^{[i+1]}\right)-g\left(\left|I_{w}^{k}\right|^{[i]}\right)},
$$

where $i$ - index pointing at successive solutions obtained using strategy $A$ sorted in ascending order in accordance with the maximum value of $T H D V_{w},\left|I_{w}^{k}\right|^{[i]}-$ RMS value of the compensator current for the solution number $i$.

The function $g(\cdot)$ is discontinuous as the cost of compensators is constant if their required currents fall between two adjacent nominal currents. Exemplary relation between the cost and the nominal current on the base of price lists of APFs available at the moment in the market as well as the function $g(\cdot)$, which corresponds to this relation, have been shown in Fig. 2.

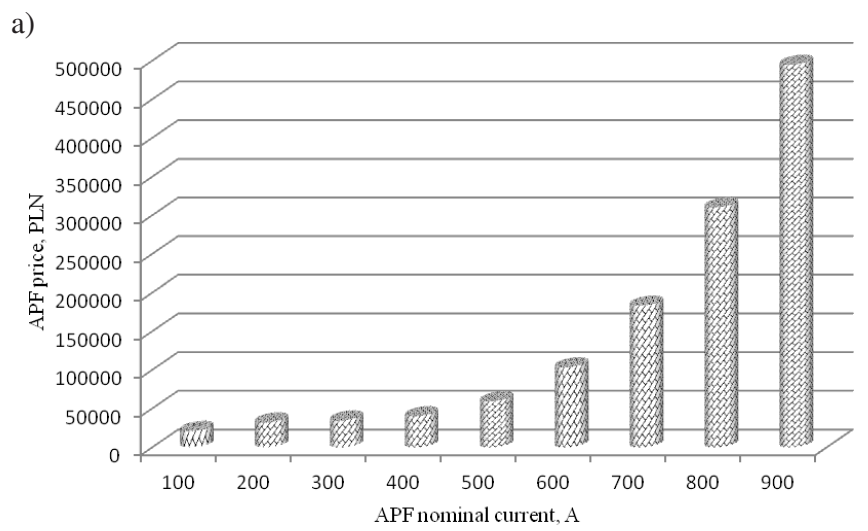

b)

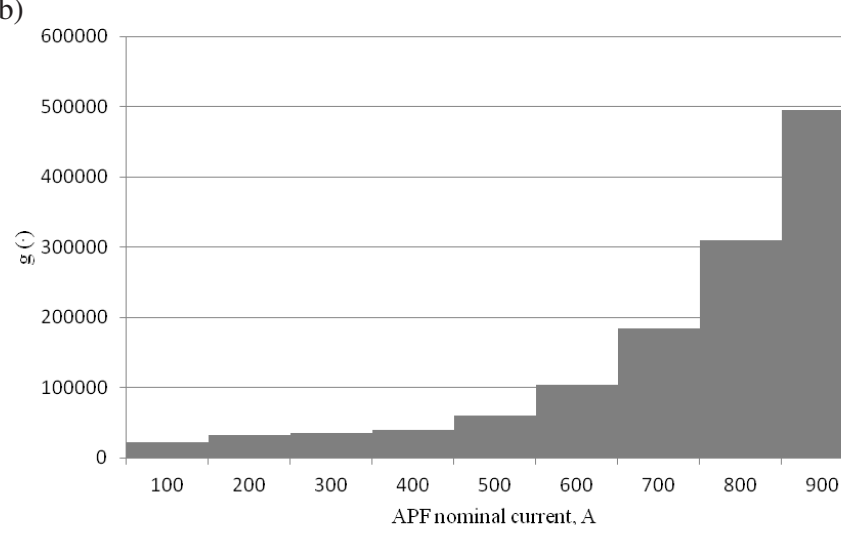

Fig. 2. Exemplary relation between compensator price and its nominal current (a), the corresponding function $g(\cdot)$ (b) 
Some other optimization approaches are also possible, e.g. minimization of total power losses in the power system. The comparative study of a few goal functions, which could be applied in optimization of compensator sizing and placement, has been carried out in [22].

The optimization of compensator sizing and placement requires analysis of many combinations of APFs connection to the system buses using one of numerous methods. In this paper combinatorial approach combined with analysis from the point of view of power quality and cost effectiveness has been used. As a result it is possible to minimize the total cost and determine the number of compensators and their nominal currents as well as the buses to which they should be connected in order to achieve the goal. The cost of APFs is not minimized directly if the goal function is based on THDI coefficients - see Eq. (1). However, as it has been discussed above the economical criterion can also be taken into account when selecting the best solution. The other way to take the cost into consideration consists in minimization of the RMS values of the compensator currents or a function based on arguments representing these values [17]. In the approach used in this paper the focus is set on elimination of current higher harmonics in given power lines [24] and as a result also reducing supplying voltage higher harmonics. The computational complexity of the proposed method is proportional to the number of buses to which compensators can be connected, but the calculation time is relatively short comparing with other approaches [30] and so it is possible to get solutions for even large power systems.

\section{Optimization algorithm}

The optimization algorithm used to achieve the goal has been presented in Fig. 3.

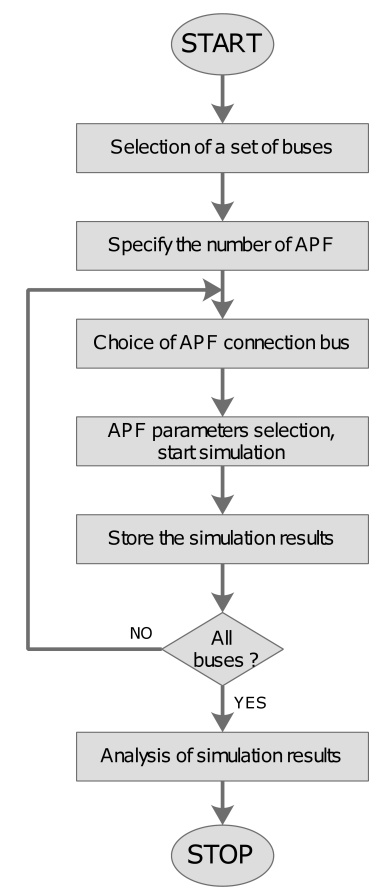

Fig. 3. Algorithm of optimization of EFA allocation
The first step consists in determination of a set of buses $W_{s}$ to which APFs can be connected. It has been assumed in accordance with the common industrial practice that it comprises buses in which there are nonlinear loads. In the case of the test system under consideration this set consists of the buses with the following numbers:

$$
W_{s}=\{8,9,10,11,12,13,14,15\} .
$$

The number of APFs is set in the second step of the algorithm and the proper combinatorial optimization process starts. All possible combinations for the given number of compensators and the set (4) are analyzed. The last steps consist in aggregation of optimization results for a given number of compensators followed by the evaluation of the results. Finally, the optimum solution for the assumed strategy is chosen.

\section{Optimization results}

The results obtained for the first described optimization strategy (A) have been presented in Fig. 4. It shows comparison of the THDV coefficients after optimization for the number of APFs from 1 to 8 . Each presented value is a maximum obtained for a given number of APFs, i.e. for the other buses belonging to the particular solution the $T H D V$ values are lower.

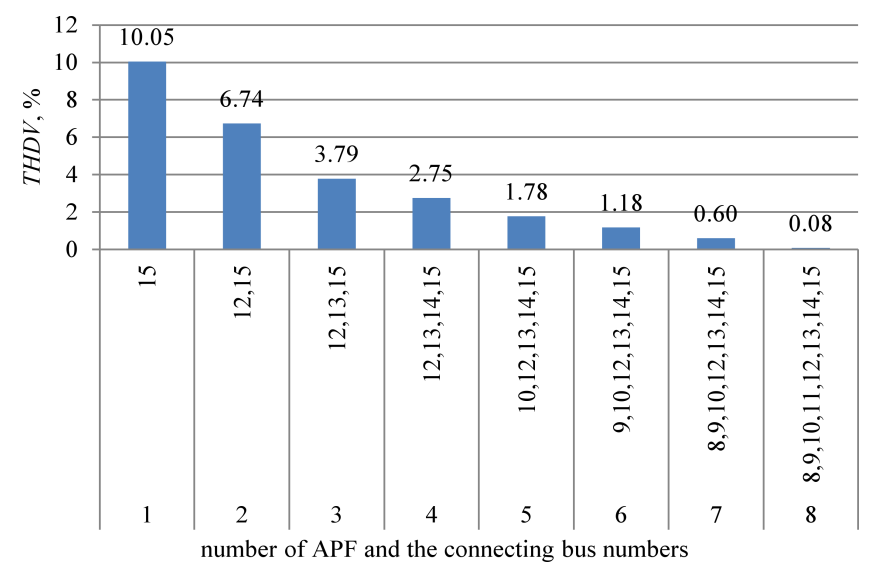

Fig. 4. Comparison of the minimum values of $T H D V$ in the chosen bus of the system for variable number of compensators (from 1 to 8 ) and different connection arrangements (the numbers of buses are given) - optimization strategy (A)

It is evident that the best solution is reached if the number of APFs is equal to the number of nonlinear loads. In this case a state, which can be called a total compensation, is obtained. It means that all unwanted current components flow only in the circuit loops consisting of APFs and nonlinear loads and so they do not influence the supplying power network at all [31]. The THDV coefficients for the case of total compensation ( 8 APFs) have been presented in Table 1 in a row labeled as optimization strategy $A$. The values written using italic font correspond to the buses in which APFs have been connected. 
Table 1

$T H D V$ coefficients for the system buses before and after optimization using three strategies

\begin{tabular}{|c|c|c|c|c|c|c|c|c|c|c|c|c|c|c|c|c|c|}
\hline \multirow{3}{*}{$\begin{array}{l}\text { Optimization } \\
\text { strategy }\end{array}$} & \multicolumn{17}{|c|}{ Bus name and number } \\
\hline & $\begin{array}{c}\text { Sub } \\
12.47 \mathrm{kV}\end{array}$ & $\begin{array}{c}\text { Near } \\
\text { Sub S. }\end{array}$ & $\begin{array}{c}\text { Near } \\
\text { Sub N. }\end{array}$ & PBS & $\mathrm{PBN}$ & Base & Star & Wilderness & Dorsey & Taylor & Longs & Apollo & Jupiter & WipeOut & BigBoss & Shop & $\begin{array}{c}\text { Sub } \\
138 \mathrm{kV}\end{array}$ \\
\hline & 1 & 2 & 3 & 4 & 5 & 6 & 7 & 8 & 9 & 10 & 11 & 12 & 13 & 14 & 15 & 16 & 20 \\
\hline $\begin{array}{c}\text { No } \\
\text { optimization }\end{array}$ & 11.3 & 11.4 & 11.4 & 11.7 & 11.7 & 11.9 & 12.4 & 12.5 & 12.4 & 12.4 & 12.5 & 13.1 & 12.8 & 12.7 & 13.0 & 12.4 & 3.3 \\
\hline $\mathrm{A}$ & 0.07 & 0.07 & 0.07 & 0.07 & 0.07 & 0.07 & 0.08 & 0.08 & 0.08 & 0.08 & 0.08 & 0.08 & 0.08 & 0.08 & 0.08 & 0.08 & 0.02 \\
\hline B & 4.18 & 4.22 & 4.22 & 4.30 & 4.30 & 4.38 & 4.55 & 4.64 & 4.59 & 4.59 & 4.68 & 5.04 & 4.77 & 4.66 & 4.61 & 4.56 & 1.22 \\
\hline $\mathrm{C}$ & 5.37 & 5.78 & 5.78 & 5.89 & 5.89 & 5.99 & 6.24 & 6.36 & 6.27 & 6.28 & 6.37 & 6.89 & 6.61 & 6.40 & 6.32 & 6.26 & 1.68 \\
\hline
\end{tabular}

The results for the second optimization strategy (B) have been shown in Fig. 5 - for some cases the constraint (2) has not been fulfilled. The presented THDV values are the maximum ones for the selected optimum solution for each number of compensators. The bar for the case which ensures fulfillment of the power quality constraint and reduction of the cost (the number of APFs is the smallest comparing with the other cases for which the constraint is also fulfilled) is striped. The $T H D V$ coefficients obtained in all the buses of the system for the chosen optimum case (4 APFs in buses 10, 13, 14 and 15) have been presented in Table 1. The values corresponding to the buses in which APFs have been connected are written using italic font like for the first strategy.

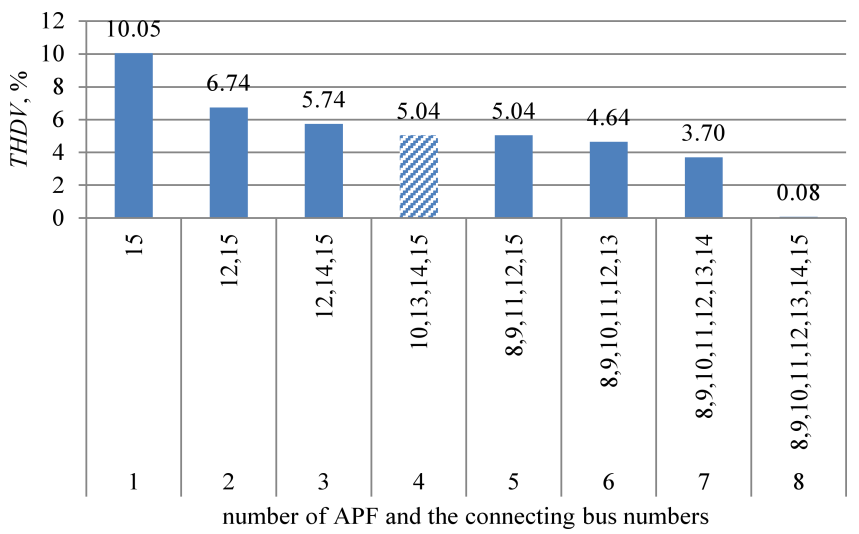

Fig. 5. Comparison of $T H D V$ values in the chosen bus of the system for variable number of compensators (from 1 to 8 ) and different connection arrangements (the numbers of buses are given) - optimization strategy (B)

In the case of the third strategy (C) the optimum solution should ensure a compromise between power quality improvement and the cost of the compensation. In order to follow this approach the maximum values of the $T H D V$ coefficients before and after compensation have been compared keeping in mind the cost of the compensators (see Fig. 2) required to reach this state. The results have been presented in Fig. 6 .

The solution which enables to achieve the best price/effects ratio has been pointed up in Fig. 6. The values of THDV coefficients for all the buses in this case (5 APFs in buses 8, 9, 10, 14 and 15) have been collected in Table 1. The optimum location of the APFs has been indicated as for the other strategies.

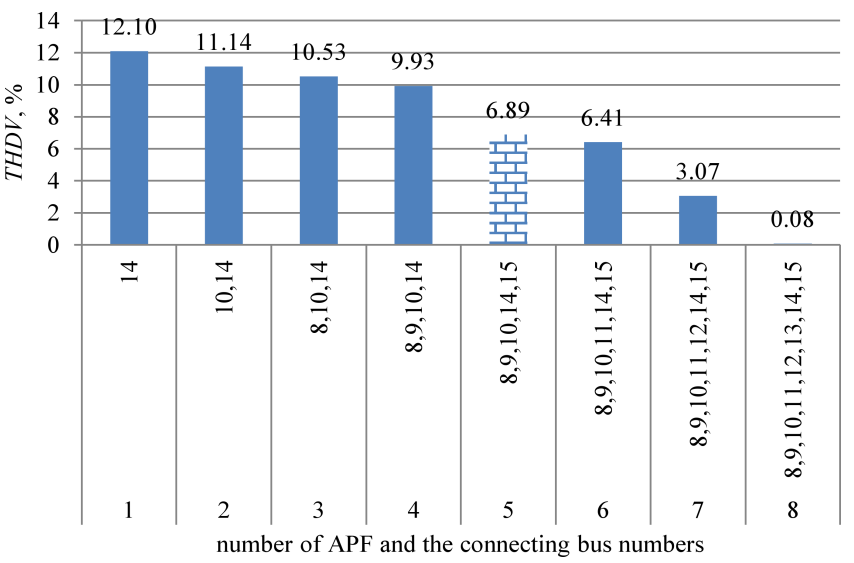

Fig. 6. Comparison of $T H D V$ values in the chosen bus of the system for variable number of compensators (from 1 to 8 ) and different connection arrangements (the numbers of buses are given) - optimization strategy $(\mathrm{C})$

The comparison has been performed not only for the coefficients determined in the frequency domain but also for time waveforms. Exemplary voltage waveforms for the node 7 and current waveforms for the line 6-7 obtained for all three optimization strategies have been shown in Figs. 7, 8 and 9. The point, for which voltages and currents have been presented, has been chosen as it supplies relatively large number of nonlinear loads.

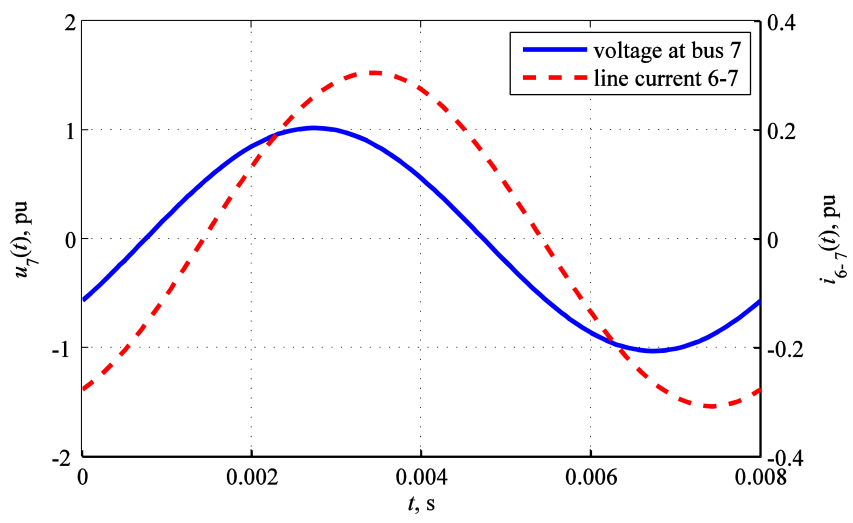

Fig. 7. Exemplary voltage (bus 7) and current (line 6-7) waveforms obtained in PCFLO - optimization strategy (A) 


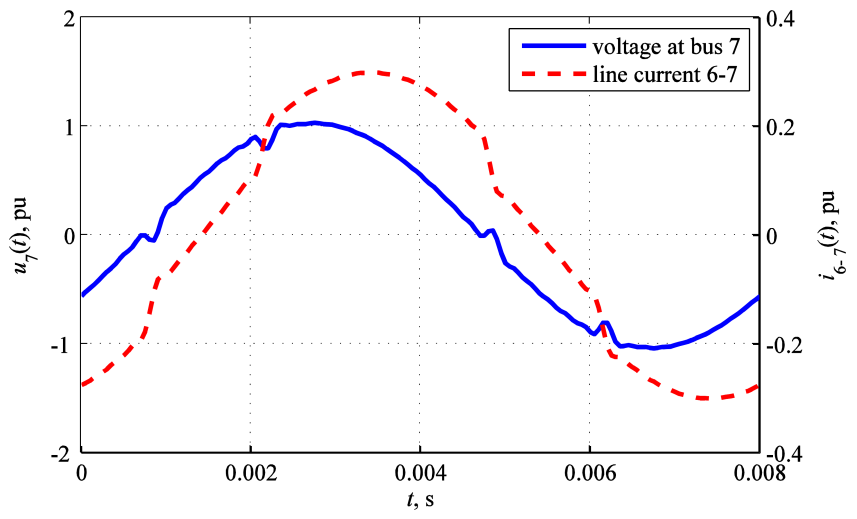

Fig. 8. Exemplary voltage (bus 7) and current (line 6-7) waveforms obtained in PCFLO - optimization strategy (B)

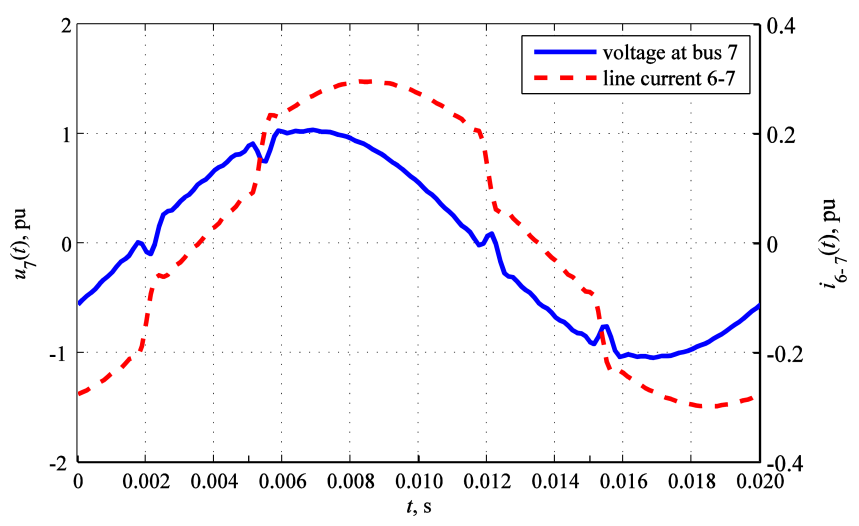

Fig. 9. Exemplary voltage (bus 7) and current (line 6-7) waveforms obtained in PCFLO - optimization strategy (C)

The comparison of costs for each solution has also been done. Fig. 10 shows relative costs. The cost of total compensation (APFs connected in all buses with nonlinear loads) has been chosen as a point of reference.

It is evident that the solution which reduces the THDV coefficients to the level imposed by standards is $40 \%$ cheaper than the total compensation and the best solution from the economical point of view is even $60 \%$ cheaper (the THDV coefficients have been reduced by half but they are above the recommended threshold). The choice of the solution depends on the particular objectives which should be achieved.

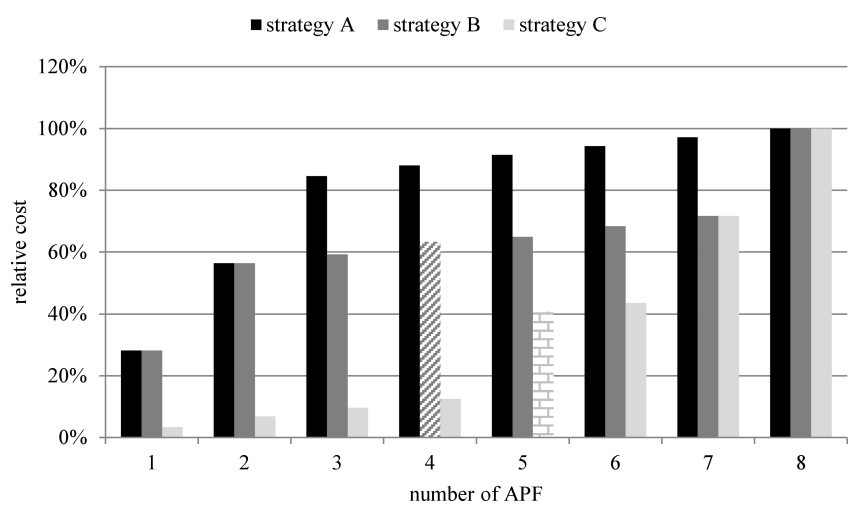

Fig. 10. Comparison of relative costs of compensation for different numbers of compensators and different assessment criteria

\section{Conclusions}

The way in which compensators are placed in the power system influences both the compensation quality and the financial cost. The optimization algorithms could be applied to find the best solution assuming one of the optimization strategies. This paper shows that minimization of the compensator currents could lead to suboptimal solutions from the economical point of view and in fact a function $g(\cdot)$ reflecting the relation between the compensator size and its price must be incorporated into objective functions.

The obtained results have been additionally verified using a model of the test system implemented in Matlab/Simulink with SimPowerSystem toolbox. The results obtained using PCFLO are consistent with those obtained in Matlab/Simulink - the comparative analysis can be found in [30]. The small differences are caused by different analysis domains (for PCFLO it is the frequency domain while for Matlb/Simulink the time domain).

Although the proposed methods are not solutions ready for immediate implementation, they can be treated as a reference point for further research and indicate possible future development directions leading to industrial solutions.

Acknowledgements. This work was supported by the Polish Ministry of Science and Higher Education under the project number N N510 257338.

\section{REFERENCES}

[1] H. Akagi, "Modern active filters and traditional passive filters", Bull. Pol. Ac.: Tech. 54 (3), 255-269 (2006).

[2] G. Benysek, M.P. Kaźmierkowski, J. Popczyk, and R. Strzelecki, "Power electronic systems as a crucial part of Smart Grid infrastructure - a survey", Bull. Pol. Ac.: Tech. 59 (4), 455-473 (2011).

[3] G.W. Chang, S.-Y. Chu, and H.L. Wang, "A new method of passive harmonic filter planning for controlling voltage distortion in a power system", IEEE Trans. on Power Delivery 21 (1), 305-312 (2006).

[4] R. Dehini and S. Sefiane, "Power quality and cost improvement by passive power filters synthesis using ant colony algorithm", J. Theoretical and Applied Information Technology 23 (2), 7079 (2011).

[5] G. Carpinelli, G. Ferruzzi, and A. Russo, "Trade-off analysis to solve a probabilistic multi-objective problem for passive filtering system planning", Int. J. Emerging Electric Power Systems 14 (3), 275-284 (2013).

[6] N. He, D. Xu, and L. Huang, "The application of particle swarm optimization to passive and hybrid active power filter design", IEEE Trans. on Industrial Electronics 56 (8), 28412851 (2009).

[7] Y.-Y. Hong and Y.-K. Chang, "Determination of locations and sizes for active power line conditioners to reduce harmonics in power systems", IEEE Trans. on Power Delivery 11 (3), 1610-1617 (1996).

[8] R. Keypour, H. Seifi, and A. Yazdian-Varjani, "Genetic based algorithm for active power filter allocation and sizing", Electric Power Systems Research 71, 41-49 (2004).

[9] D.F.U. Ramos, J. Cortes, H. Torres, L.E. Gallego, A. Delgadillo, and L. Buitrago, "Implementation of genetic algorithms in 
ATP for optimal allocation and sizing of active power line conditioners", Proc. IEEE/PES Transmission \& Distribution Conf. and Exposition 1, 1-5 (2006).

[10] N. Dehghani and I. Ziari, "Optimal allocation of APLCs using genetic algorithm", Proc. 43rd Int. Universities Power Engineering Conference UPEC 1, 1-4 (2008).

[11] Wang Yan-Song, Shen Hua, Liu Xue-min, Liu Jun, Gou Songbo, "Optimal allocation of the active filters based on the TABU algorithm in distribution network", Proc. Int. Conf. on Electrical and Control Engineering ICECE 1, 1418-1421 (2010).

[12] C.S. Gehrke, A.M.N. Lima, and A.C. Oliveira, "Evaluating APLCs placement in a power system based on real-time simulation", 2012 IEEE Energy Conversion Congress and Exposition 2011, CD-ROM (2012).

[13] A. Moradifar and H.R. Soleymanpour, "A fuzzy based solution for allocation and sizing of multiple active power filters", J. Power Electronics 12 (5), 830-841 (2012).

[14] I. Ziari and A. Jalilian, "A new approach for allocation and sizing of multiple active power-line conditioners", IEEE Trans. on Power Delivery 25 (2), 1026-1035 (2010).

[15] I. Ziari and A. Jalilian, "Optimal placement and sizing of multiple APLCs using a modified discrete PSO”, Int. J. Electrical Power and Energy Systems 43 (1), 630-639 (2012).

[16] K. Kennedy, G. Lightbody, R. Yacamini, M. Murray, and J. Kennedy, "Online control of an APLC for network-wide harmonic reduction", IEEE Trans. on Power Delivery 21 (1), 432439 (2006).

[17] D. Grabowski, M. Maciążek, and M. Pasko, "Sizing of active power filters using some optimization strategies", Int. J. for Computation and Mathematics in Electrical and Electronic Engineering COMPEL 32 (4), 1326-1336 (2013).

[18] C.S. Gehrke, A.M.N. Lima, and A.C. Oliveira, "Cooperative control for active power compensators allocated in distributed networks", 2012 IEEE Energy Conversion Congress and Exposition 1, 2764 (2012).

[19] H. Yue, G. Li, M. Zhou, K. Wang, and J. Wang, "Multiobjective optimal power filter planning in distribution network based on fast nondominated sorting genetic algorithms", DRPT 2011 - 2011 4th Int. Conf. on Electric Utility Deregulation and Restructuring and Power Technologies 1, 234 (2011).
[20] S.M.R. Rafiei, M.H. Kordi, G. Griva, and H. Yassami, "Multiobjective optimization based optimal compensation strategies study for power quality enhancement under distorted voltages", IEEE Int. Symp. on Industrial Electronics 1, 3284 (2010).

[21] E. González-Romera, E. Romero-Cadaval, S. Ruíz-Arranz, and M. Milanés-Montero, "Overall power quality correction in distribution networks by active power filters. optimization of location and strategy", Electrical Engineering Review 88 (1 A), 51-55 (2012).

[22] D. Grabowski and J. Walczak, "Strategies for optimal allocation and sizing of active power filters", Proc. 11-th Int. Conf. on Environment and Electrical Engineering EEEIC 1, 1-4 (2012).

[23] K. Mikołajuk and M. Dzieciątko, "The Boltzmann machine algorithm for combinatorial optimization problems", Electrical Engineering Review 78 (12), 358-362 (2002).

[24] M. Maciążek, "Power theories applications to control active compensators", Power Theories for Improved Power Quality, Springer Power Systems Series 1, 49-116 (2012).

[25] Task Force on Harmonics Modeling and Simulation, "Test systems for harmonics modeling and simulation", IEEE Trans. on Power Delivery 14, 579-585 (1999).

[26] Task Force on Harmonics Modeling and Simulation, "The modeling and simulation of the propagation of harmonics in electric power networks. Part II: Sample systems and examples", IEEE Trans. on Power Delivery 11 (1), 466-474 (1996).

[27] R. Christie, Power Systems Test Case Archive, http://www.ee.washington.edu /research/pstca/ (1993).

[28] W.M. Grady, PCFLO v6 Users' Manual, http://users.ece.utexas.edu/ grady/ (2010).

[29] IEEE Std 519-1992 IEEE Recommended Practices and Requirements for Harmonic Control in Electric Power Systems.

[30] D. Buła, D. Grabowski, M. Lewandowski, M. Maciążek, M. Pasko, A. Piwowar, and J. Walczak, Analysis and Optimization of Active Power Filter Allocation, The Publishing House of The Silesian University of Technology, Gliwice, 2013.

[31] M. Lewandowski, M. Maciążek, and D. Grabowski, "Integration of Matlab and PCFLO for harmonic flow analysis in a power system containing APF", Proc. XXXIV Int. Conf. on Fundamentals of Electrotechnics and Circuit Theory ICSPETO 1, 89-90 (2011). 